Background: Systemic lupus erythematosus (SLE) is a multisystemic autoimmune disease with heterogenous and variable behavior. The frequency of pulmonary involvement ranges from $10-90 \%$, with this variability in occurrence attributed to differences in populations and heterogeneity in detection methods and study designs. Objectives: To analyze survival in patients with SLE and the presence of pulmonary manifestations according to clinical and demographic characteristics in a cohort of patients in Colombia

Methods: Observational-analytical, retrospective, follow-up study of a cohort of adult patients with SLE. We included 559 adult patients who fulfilled the 2012 SLICC SLE classification criteria and with at least 6 months of disease evolution, treated in a medical center specialized in rheumatology with locations in 6 cities in Colombia between 2015 and 2018. Pregnant patients and those who had incomplete data or visits were excluded. Immunoserological profile was characterized and pulmonary involvement was followed for 1 year. The diagnosis of pulmonary involvement was obtained from the rheumatology report in the clinical chart. The prevalence of pulmonary manifestations and the immunoserological profile were determined and subsequently a logistic regression adjusted for age, sex and level of education was performed to establish the association between pulmonary manifestations and a positive autoantibody profile

Results: Median age was $44.5 \pm 14$ years, $96.6 \%$ were women. In patients with pulmonary compromise $9.7 \%$ were smokers, $12.4 \%$ had an active alcohol intake, $58.4 \%$ had a low socioeconomic status, $38.1 \%$ medium and $4.7 \%$ high. Pulmonary involvement was present in $20 \%$ of patients, the most common manifestations were pleuritis (14.3\%), lupus pneumonitis (3.6\%), pulmonary hypertension (3.2\%), interstitial lung disease $(2.3 \%)$, pulmonary embolism $(2.3 \%)$, pulmonary fibrosis $(2.14 \%)$, alveolar hemorrhage $(1.4 \%)$, shrinking lung $(0.2 \%)$. there was no significant difference in the follow up at one year. Immunoserological characteristics shows ANA positivity in $92 \%$, anti-DNA in $53 \%$, ENAS in $97 \%$, with anti-RNP being the most common (41.7\%), and low complement levels in $53 \%$. Mortality for pulmonary compromise was $1.4 \%$, and survival at 2 years did not have a statistically significant difference $(p=0.155)$

Conclusion: Survival was not found to be lower in patients with SLE and pulmonary involvement $(p=0.155)$ when compared to SLE patients without pulmonary involvement after 2-year follow-up. This finding was independent of age, gender, and educational level. Previous large cohorts like GLADEL and RELESSER describe an association between pulmonary involvement and lower survival HR 2,79 (95\% Cl: 1,80-4,31), $\mathrm{p}<0,001$ and HR 3.13, (95\% IC: $1.56-6.28, \mathrm{P}=0.00$ respectively. In the RELESSER cohort pleural involvement and pulmonary embolism was found to have low to minimal impact on survival.

In our cohort pleuritis was the most common finding whereas the other pulmonary manifestations were reported in less than $4 \%$ of patients. This difference could explain our differences with other cohorts. Other causes for this difference are a smaller sample size and a short follow-up.

The results of our study do not suggest that there is an association between pulmonary involvement and mortality in Colombian patients with SLE during a 2-year follow-up. We will continue to monitor and report in this regard REFERENCES:

[1] Narváez J, Borrell H, Sánchez-Alonso F, Rúa-Figueroa I, López-Longo FJ, Galindo-Izquierdo $\mathrm{M}$, et al. Primary respiratory disease in patients with systemic lupus erythematosus. (RELESSER) cohort. Arthritis Res Ther. 2018;20(1):1-10.

[2] Keane MP, Lynch JP. Pleuropulmonary manifestations of systemic lupus erythematosus. Vol. 55, Thorax. Thorax; 2000. p. 159-66.

[3] Haye Salinas MJ, Caeiro F, Saurit V, Alvarellos A, Wojdyla D, Scherbarth $\mathrm{HR}$, et al. Pleuropulmonary involvement in patients with systemic lupus erythematosus from a Latin American inception cohort (GLADEL). Lupus. 2017;26(13):1368-77

Disclosure of Interests: None declared

DOI: 10.1136/annrheumdis-2021-eular.3428

\section{AB0336 PULMONARY MANIFESTATIONS IN A COLOMBIAN COHORT OF PATIENTS WITH SYSTEMIC LUPUS ERYTHEMATOSUS}

S. Herrera ${ }^{1}$, J. C. Diaz-Coronado ${ }^{1,2}$, S. Monsalve ${ }^{2}$, S. Guerra-Zarama ${ }^{2}$, M. F. Saavedra Chacón ${ }^{2}$, J. Barbosa' ${ }^{1}$, J. D. Serna Giraldo², J. D. Lopez ${ }^{2}$, J. M. Gutiérrez ${ }^{2}$, T. Vega' ${ }^{2}$, D. Orozco ${ }^{2}$, D. Ocampo ${ }^{2}$, N. Zuluaga ${ }^{2}$, D. HernandezParra $^{1}$, D. Rojas-Gualdrón ${ }^{3}$, R. Pineda.Tamayo ${ }^{1} .{ }^{1}$ Artmedica IPS, Clinical Information Group, Medell in, Colombia; ${ }^{2}$ CES University, Department of Internal Medicine, Medellín, Colombia; ${ }^{3}$ CES University, Facultad de medicina, Medellín, Colombia

Background: Pulmonary manifestations are frequent in systemic lupus erythematosus (SLE) with a frequency of $30-90 \%$ that depends on the cohort and the methods used for their identification. The association of this compromise with mortality highlights its importance and the need for biomarkers to adequately predict this complication. We describe the prevalence of pulmonary manifestations, and the clinic and immunoserological characteristics of 551 Colombian patients with SLE
Objectives: We performed an observational and analytic study of a retrospective cohort with adult SLE patients who fulfilled the 2012 SLICC classification criteria and that had a history of at least 6 months of the disease. These patients were treated in a specialized center of rheumatology with presence in six cities of Colombia between 2015 and 2018. We excluded pregnant patients and those with incomplete data for our survey. The first clinic consult occurred between 2015 and 2018, being defined as moment one. The follow up one year later was defined as moment two. We obtained 710 registries that were potentially eligible and analyzed 465 patients at moment two after applying the exclusion criteria Methods: In 465 eligible patients, $20,5 \%$ had pulmonary compromise $193.8 \%$ female) with a median age of 42,4 years. The average SLICC Damage Index of 551 patients with SLE was 0,9 in women and 1.05 in men, while the average SDI of patients with pulmonary compromise was 1 . The most frequent manifestation was pleural $(14.3 \%)$, followed by Lupus pneumonitis (3.6\%) and pulmonary hypertension (3.2\%). Other manifestations and serological characteristics are recorded in Table 1. Of note, ANA homogeneous pattern was the most common (34.5\%), anti-RNP positivity was $41.7 \%$, anti-dsDNA positivity was $53.1 \%$ and $53.1 \%$ had hypocomplementemia.

Results: The prevalence of pulmonary manifestations in our cohort was $20,5 \%$, which is lower that in the previous described GLADEL cohort $(28,4 \%)$. This could be explained by the regional differences of ethnicities in Latin America and in immune-serological profiles. Anti-RNP positivity was frequent (41.7\%) and new pulmonary compromise for one year follow-up was rare. Of not, the mean damage index for our patients with pulmonary manifestations was 1 , this could highlight the importance of this organ as a causa of higher damage accrual and mortality, which we will explore in the future

Conclusion: The prevalence of pulmonary manifestations in our cohort was $20,5 \%$, which is lower that in the previous described GLADEL cohort $(28,4 \%)$ This could be explained by the regional differences of ethnicities in Latin America and in immune-serological profiles. Anti-RNP positivity was frequent (41.7\%) and new pulmonary compromise for one year follow-up was rare. Of not, the mean damage index for our patients with pulmonary manifestations was 1 , this could highlight the importance of this organ as a causa of higher damage accrual and mortality, which we will explore in the future

\section{REFERENCES:}

[1] G. Aguilera-Pickens, C. Abud-Mendoza. Pulmonary Manifestations in Systemic Lupus Erythematosus: Pleural Involvement, Acute Pneumonitis, Chronic Interstitial Lung Disease and Diffuse Alveolar Hemorrhage. Reumatol Clin. 2018;14(5):294-300.

[2] Haye Salinas MJ, Caeiro F, Saurit V. Pleuropulmonary involvement in patients with systemic lupus erythematosus from a Latin American inception cohort (GLADEL). Lupus (2017) 0, 1-10.

[3] Santamaria-Alza Y, Sanchez-Bautista J, Fajardo-Rivero J. Acute respiratory involvement in Colombian patients with systemic lupus erythematosus undergoing chest computed tomography. Int J Rheum Dis. 2019;00:1-7.

Table 1. clinical and immunoserological characteristics

\begin{tabular}{|c|c|c|c|}
\hline & & $\mathbf{n}$ & $\%$ \\
\hline \multicolumn{2}{|l|}{ Women } & 106 & 93,8 \\
\hline \multicolumn{2}{|l|}{ Global mortality } & 46 & 8,3 \\
\hline \multicolumn{2}{|c|}{ Pulmonary compromise mortality } & 8 & 7,1 \\
\hline \multicolumn{2}{|c|}{ ANA } & 104 & 92 \\
\hline \multicolumn{2}{|l|}{ Anti-DNA } & 60 & 53,1 \\
\hline \multicolumn{2}{|l|}{ ENAS } & & 97,2 \\
\hline \multicolumn{2}{|l|}{ Ro } & $35 / 87$ & 40,2 \\
\hline \multicolumn{2}{|l|}{ La } & $14 / 85$ & 16,5 \\
\hline \multicolumn{2}{|l|}{ SM } & $32 / 88$ & 36,4 \\
\hline \multirow[t]{2}{*}{ RNP } & & $35 / 84$ & 41,7 \\
\hline & Follow up $1 \%$ & Follow up $2 \%$ & $P$ Value * \\
\hline Pulmonary hypertension & 3,2 & 2,8 & 0,28 \\
\hline Pulmonary fibrosis & 2,14 & 2,6 & 1 \\
\hline Shrunken lung & 0,2 & 0,2 & 1 \\
\hline Pleuritis & 14,3 & 15,05 & 0,42 \\
\hline Lupus pneumonitis & 3,6 & 3,01 & 0,85 \\
\hline Alveolar hemorrhage & 1,4 & 1,3 & 0,76 \\
\hline Pulmonary embolism & 2,3 & 1,93 & 0,72 \\
\hline
\end{tabular}

Disclosure of Interests: None declared

DOI: 10.1136/annrheumdis-2021-eular.3452

\begin{tabular}{|l|l|}
\hline AB0337 & ASSESSMENT OF HEALTH-RELATED QUALITY OF \\
LIFE IN SYSTEMIC LUPUS ERYTHEMATOSUS USING \\
THE SLEQOL (SYSTEMIC LUPUS ERYTHEMATOSUS- \\
SPECIFICQUALITY OF LIFE QUESTIONNAIRE)
\end{tabular}

M. Brahem ${ }^{1}$, M. Ardhaoui ${ }^{1}$, S. Arfa ${ }^{2}$, H. Hachfi ${ }^{1}$, B. Ben Rejeb ${ }^{2}$, R. Sarraj ${ }^{1}$, M. Hassayoun ${ }^{1}$, S. Abdellatif ${ }^{1}$, A. Ben Salem ${ }^{1}$, R. Ben Tekaya ${ }^{1}$, O. Berriche ${ }^{2}$, Y. Mohamed ${ }^{1} .{ }^{1}$ Taher Sfar Hospital, Rheumatology, Mahdia, Tunisia; ${ }^{2}$ Taher Sfar Hospital, Internal Medicine, Mahdia, Tunisia 